\title{
Cardiorespiratory Fitness After A 12-Week Cardiac Rehabilitation Program - Insights in a Contemporary Cohort of Cardiac Patients
}

\author{
Masárová $\mathbf{V}^{1 *}$, Haegele $\mathbf{M}^{1}$, Frey $\mathrm{S}^{1}$, Schaerli $\mathrm{N}^{2}$, Burkard $\mathrm{T}^{2}$, Lange $\mathrm{R}^{2}$ and Pfister $\mathbf{0}^{1,2}$ \\ ${ }^{1}$ Cardiovascular Research Institute Basel, University Hospital Basel, Switzerland \\ ${ }^{2}$ Department of Cardiology, University Hospital Basel, Switzerland
}

*Corresponding author: Veronica Masárová, Cardiovascular Research Institute Basel, University Hospital Basel, Basel, Switzerland

To Cite This Article: Masárová V, Haegele M, Frey S, Schaerli N, Burkard T, Lange R, Pfister O. Cardiorespiratory Fitness After A 12-Week Cardiac Rehabilitation Program - Insights in a Contemporary Cohort of Cardiac Patients. Am J Biomed Sci \& Res. 2021 - 13(1). AJBSR.MS.ID.001823. DOI: 10.34297/AJBSR.2021.13.001823.

Received: 鮆 April 23, 2021; Published: 眥 May 24, 2021

\section{Abstract}

Purpose: Cardiorespiratory fitness (CRF) is a strong predictor of mortality. Cardiac rehabilitation (CR) increases CRF measured by peakVO2 by an average of $2 \pm 3.3 \mathrm{ml} / \mathrm{kg} / \mathrm{min}$ according to historical data. Progress in interventional and surgical procedures as well as advances in medical therapies and CR training protocols may result in optimization of CRF gain after a phase 2 CR program. Because data on CRF in contemporary patient populations undergoing CR are scarce, we here report CRF data from our latest patient cohort.

Methods: From January to December 2018 all consecutive patients undergoing a 12-week ambulatory CR program at our institution were included in this analysis. CRF was determined by cardiopulmonary exercise testing. Improvement in CRF was measured as the difference in oxygen uptake at the anaerobic threshold (VO2@AT) and at exhaustion (peakVO2) before and after CR.

Results: 417 predominantly male patients (83\% male), mean age 61.5 (SD 12.5) years, completed the 12-week CR program. The majority of patients were included after an acute coronary syndrome (ACS: 65\%), followed by patients with chronic coronary syndromes (CCS: 20\%), heart failure (7.5\%) or valve disease (7.5\%). Mean gain of increased CRF was 3,3ml/kg/min (SD 6.5) (VO2@AT) (p=0.0001) and 3,2ml/kg/min (SD 7.0) (VO2 max) ( $\mathrm{p}=0.0001)$. There was no difference between men and women ( $\mathrm{p}=0.127$ V02@AT, $\mathrm{p}=0.156$ V02 max respectively), however, as expected, there was a statistically significant decrease in CRF improvement with increasing age $(-0.085 \mathrm{ml} / \mathrm{kg} / \mathrm{min} / \mathrm{year}, \mathrm{p}=0.001)$.

Conclusion: Though modern therapies reduce length of hospital stay and major cardiovascular events in patients after ACS, CCS and/or heart failure, participation in a 12-week ambulatory CR program still results in a clinically substantial increase in CRF regardless of sex and age. These results in a contemporary real-life-population are better than historical data and match results achieved in recent randomised prospective trials investigating high intensity interval training (HIIT)(Figure 1-3).

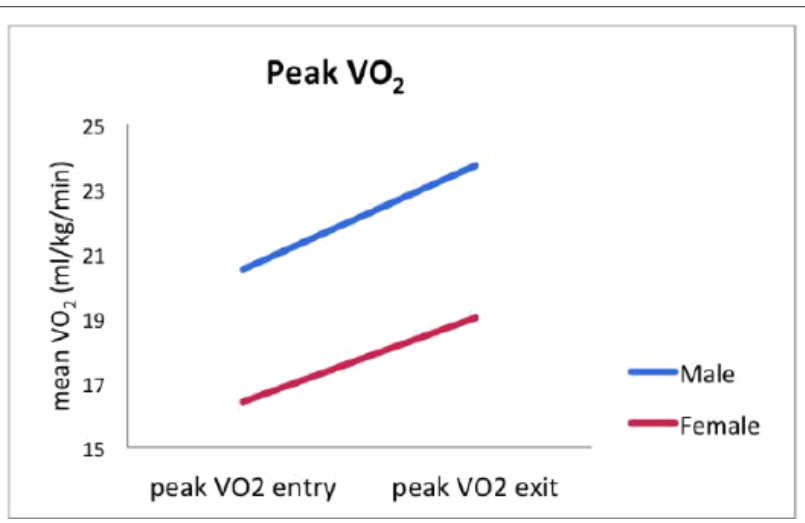

Figure 1: Increase of the peak VO2 measured before and after the 12-week rehabilitation program. 

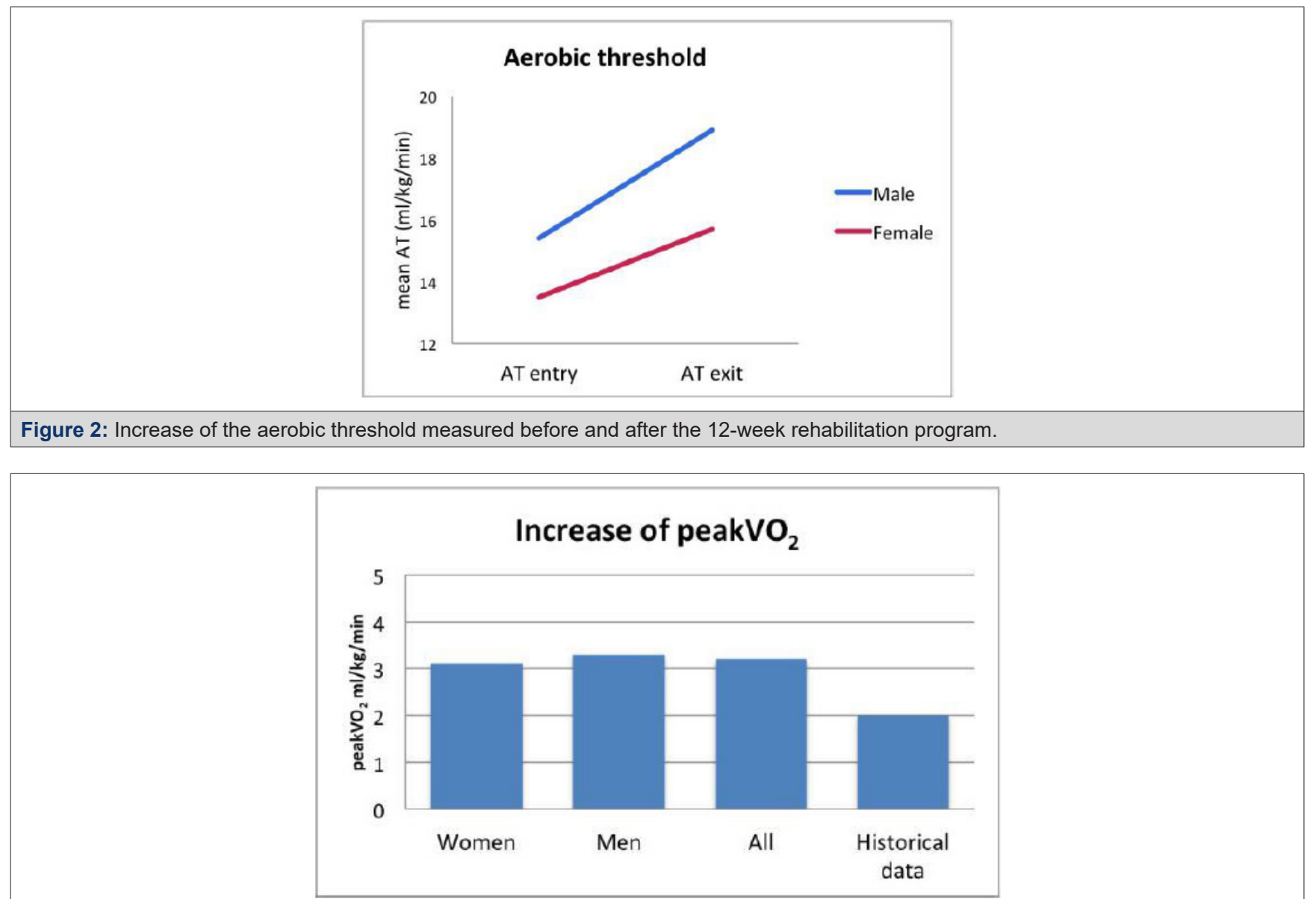

Figure 3: Improvement of CRF measured with peakVO2 $(\mathrm{ml} / \mathrm{kg} / \mathrm{min})$ of our sample compared with historical data.

Keywords: Cardiac rehabilitation; Secondary prevention; Cardiorespiratory fitness 